\title{
QUASI-LOCAL ENERGY-MOMENTUM AND THE SEN GEOMETRY OF TWO-SURFACES
}

\author{
LÁSZLÓ B. SZABADOS \\ Research Institute for Particle and Nuclear Physics \\ H-1525 Budapest 114, P.O. Box 49, Hungary \\ E-mail: lbszab@rmki.kfki.hu
}

\begin{abstract}
We review the main ideas of the two dimensional Sen geometry and apply these concepts $i$. in finding the 'most natural' quasi-local energy-momentum, $i i$. in characterizing the zero energy-momentum and zero mass configurations and iii. in finding the quasi-local radiative modes of general relativity.
\end{abstract}

1. Introduction. As a consequence of the exact diffeomorphism invariance of general relativity the familiar Lagrangian scenario $[1,2]$ does not yield reasonable energymomentum and angular momentum density for the gravitational field. More precisely, if for the gravitational field variables we choose the components $g^{a b}$ of the metric in some coordinate system $\left\{x^{a}\right\}$ then the canonical energy-momentum, the canonical spin and the canonical Noether current will be pseudotensorial; whilst if a rigid (e.g. orthonormal or complex null) tetrad field $\left\{E_{a}^{a}\right\}$ is chosen for the field variables then the canonical Noether current will be $S O(1,3)$-gauge dependent [3-5].

If however the spacetime is asymptotically flat then one can define global energymomentum, i.e. it is possible to associate an energy-momentum four-vector with the whole spacetime. Namely, if the spacetime is asymptotically flat at spacelike infinity then this four-vector is the ADM energy-momentum [6], $P_{A D M}^{a}\left[\$_{\infty}\right]$, associated with a spherical cut $\$_{\infty}$ of the spacelike infinity $\mathcal{H}$ of Beig and Schmidt $[7,8]$. If the spacetime is asymptotically flat at future null infinity then this energy-momentum is the Bondi-Sachs energy-momentum $P_{B S}^{a}\left[\$_{\infty}\right]$ associated with a spherical cut $\$_{\infty}$ of the future null infinity $\mathcal{I}^{+}$of Penrose $[9,10]$. The 2-surfaces $\$_{\infty}$ can always be considered as the intersections $\mathcal{H} \cap \Sigma$ and $\mathcal{I}^{+} \cap \Sigma$, respectively, where $\Sigma$ is a smooth spacelike hypersurface extending to

1991 Mathematics Subject Classification: Primary 0240; Secondary 0420C.

Partially supported by the Hungarian Scientific Research Fund grants OTKA 016246 and OTKA 017176 and by the exchange program of the Polish and Hungarian Academies of Sciences grant No 5-1995.

The paper is in final form and no version of it will be published elsewhere. 
spacelike/future null infinity. By the positive energy theorem [10-29] these four-vectors are future directed and timelike provided the matter fields satisfy the dominant energy condition; futhermore they are zero iff the domain of dependence $D(\Sigma)$ is flat $[18,23,24]$.

The most elegant proof of this theorem is probably that based on the use of twocomponent spinors. (A general and exhaustive introduction to the theory of two-component spinors is the volume of Penrose and Rindler [10]. Our conventions and notations are mostly those of [10]. *) To recall the basic idea let us define the Nester-Witten 2-form associated with any pair of smooth spinor fields $\lambda_{A}$ and $\mu_{A}$ by

$$
u(\lambda, \bar{\mu}):=\mathrm{i} \bar{\mu}_{A^{\prime}} \nabla_{B B^{\prime}} \lambda_{A} \mathrm{~d} x^{a} \wedge \mathrm{d} x^{b} .
$$

The importance of this complex valued 2 -form is shown by the following three facts. First, its exterior derivative takes the form

$$
\mathrm{d} u(\lambda, \bar{\mu})=-\frac{1}{2} \lambda^{A} \bar{\mu}^{A^{\prime}} G_{a b} \frac{1}{3 !} \varepsilon^{b}{ }_{c d e} \mathrm{~d} x^{c} \wedge \mathrm{d} x^{d} \wedge \mathrm{d} x^{e}+\mathrm{i} \nabla_{B B^{\prime}} \lambda_{A} \nabla_{C C^{\prime}} \bar{\mu}_{A^{\prime}} \mathrm{d} x^{a} \wedge \mathrm{d} x^{b} \wedge \mathrm{d} x^{c}
$$

The second term on the right, denoted by $\Gamma(\lambda, \bar{\mu})$, is known as Sparling's 3-form [10,30]. This equation looks like a superpotential equation in the Lagrangian field theory: the Nester-Witten 2-form is the superpotential, Sparling's 3-form corresponds to the Noether current and the pair of spinor fields as a single object is the generator the Noether current is associated with. In fact, Sparling's form can be extended to be a 3 -form on the bundle of linear frames over the spacetime manifold and the various energy-momentum pseudotensors can be recovered simply as the pull back of Sparling's form along various local sections of the linear frame bundle $[31,32]$. Second, if $\$$ is any closed, orientable smooth spacelike 2 -surface then one can define the integral

$$
H_{\$}[\lambda, \bar{\mu}]:=\frac{2}{\kappa} \oint_{\$} u(\lambda, \bar{\mu}) .
$$

It is easy to prove that $H_{\$}$ is a Hermitian scalar product on the infinite dimensional complex vector space $C^{\infty}\left(\$, \mathbf{S}_{A}\right)$ of the smooth covariant spinor fields on $\$$. Thus if $\lambda \frac{A}{A}=\left(\lambda_{A}^{0}, \lambda_{A}^{1}\right)$ is a smooth normalized spinor dyad on $\$$ then $H_{\$}\left[\lambda \underline{A}, \bar{\lambda}^{\underline{B}}\right]$ is a $2 \times 2$ Hermitian matrix. Now recall that both at spacelike and null infinity there are precisely two linearly independent constant spinor fields ${ }_{0} \lambda \frac{A}{A}=\left({ }_{0} \lambda_{A}^{0},{ }_{0} \lambda_{A}^{1}\right)$ that can be normalized by $\varepsilon^{A B}{ }_{0} \lambda \frac{A}{A}{ }_{0} \lambda \frac{B}{B}=\epsilon^{\underline{A}} \underline{B}$, where $\epsilon^{\underline{A}} \underline{B}$ is the alternating symbol. Then for any constant Hermitian matrix $K_{\underline{A}} \underline{B}^{\prime}$ the vector (or rather 1-form) field $K_{a}:=K_{\underline{A} \underline{B}^{\prime}{ }_{0}} \lambda \frac{A}{A}{ }_{0} \bar{\lambda}^{\bar{B}^{\prime}}$ is a translation at spacelike/null infinity. If we blow up our 2-surface $\$$ to tend to a spherical cut $\$_{\infty}$ of the spacelike/future null infinity such that the dyad $\lambda \frac{A}{A}$ tends to the dyad of the constant spinor fields ${ }_{0} \lambda \frac{A}{A}$ then

$$
H_{\$}\left[\lambda \underline{A}, \bar{\lambda}^{\prime} \underline{B}^{\prime}\right] \longrightarrow \begin{cases}P \frac{A}{A} \underline{B}^{\prime}{ }^{\prime}\left[\$_{\infty}\right] & \text { if } \$ \rightarrow \$_{\infty}:=\mathcal{H} \cap \Sigma \\ P \frac{A}{B} \underline{B}^{\prime}\left[\$_{\infty}\right] & \text { if } \$ \rightarrow \$_{\infty}:=\mathcal{I}^{+} \cap \Sigma .\end{cases}
$$

Third, let $\Sigma$ be a spacelike hypersurface with future directed unit timelike normal $t^{a}$. Then the pull back of Sparling's equation (1.2) along the natural injection $i: \Sigma \rightarrow M$ is

* In particular the spacetime signature is $(+---)$, the curvature and Ricci tensors are $R_{b c d}^{a} X^{b}:=-\left(\nabla_{c} \nabla_{d}-\nabla_{d} \nabla_{c}\right) X^{a}$ and $R_{b d}:=R_{b a d}^{a}$, respectively, and the curvature scalar is $R:=g^{a b} R_{a b}$. Thus Einstein's equations take the form $G_{a b}:=R_{a b}-\frac{1}{2} R g_{a b}=-\kappa T_{a b}$ with $\kappa>0$. 
equivalent to

$$
\begin{aligned}
D_{A A^{\prime}}\left(t^{B A^{\prime}} \bar{\mu}^{B^{\prime}} \mathcal{D}_{B B^{\prime}} \lambda^{A}-t^{A B^{\prime}} \bar{\mu}^{A^{\prime}} \mathcal{D}_{B B^{\prime}} \lambda^{B}\right)=-\frac{1}{2} t^{a} G_{a b} \lambda^{B} \bar{\mu}^{B^{\prime}}- \\
-h^{e f} t^{A A^{\prime}}\left(\mathcal{D}_{e} \lambda_{A}\right)\left(\mathcal{D}_{f} \bar{\mu}_{A^{\prime}}\right)-2 t^{A A^{\prime}}\left(\mathcal{D}_{A B^{\prime}} \bar{\mu}^{B^{\prime}}\right)\left(\mathcal{D}_{B A^{\prime}} \lambda^{B}\right),
\end{aligned}
$$

where $h_{a b}:=g_{a b}-t_{a} t_{b}$ is the induced metric, $D_{a}$ is the induced Levi-Civita covariant derivation on $\Sigma$ and $\mathcal{D}_{a}$ is the 3 dimensional Sen operator [33]: $\mathcal{D}_{A A^{\prime}} \lambda_{B}:=h_{a}^{e} \nabla_{e} \lambda_{B}=$ $D_{A A^{\prime}} \lambda_{B}+\chi_{A A^{\prime} B B^{\prime}} t^{B^{\prime}}{ }_{C} \lambda^{C}$. Here $\chi_{a b}$ is the extrinsic curvature of $\Sigma$. For $\mu_{A}=\lambda_{A}(1.5)$ is the Reula-Tod form [22] of the Sen-Witten identity [33], and if the matter fields satisfy the dominant energy condition then the first term on the right is positive definite, the second term is always positive definite but the third term is negative definite. Thus the right hand side of (1.5) does not have definite sign. If however $\Sigma$ extends to spacelike or null infinity then for any constant spinor ${ }_{0} \lambda^{A}$ at infinity there is a solution of the so-called Sen-Witten equation $\mathcal{D}_{A A^{\prime}} \lambda^{A}=0$ on $\Sigma$ which tends to ${ }_{0} \lambda^{A}$, furthermore the integral for $\$_{\infty}$ of the left hand side of (1.5) becomes $H_{\$_{\infty}}\left[{ }_{0} \lambda,{ }_{0} \bar{\lambda}\right]$, yielding a nonnegativity proof of the ADM and Bondi-Sachs energies [22]. The key object in this non-negativity proof is therefore the Sen operator $\mathcal{D}_{a}$. This operator is not an intrinsic object on $\Sigma$, it depends on the imbedding of $\Sigma$ in $M$ too.

Finally we can speak about gravitational energy-momentum at the quasi-local level, i.e. between the two extrems, the density and the total energy-momentum. The idea is that we associate energy-momentum with extended but finite pieces of the spacetime manifold. In contrast to the ADM or Bondi-Sachs energy-momentum, however, there is not known any generally accepted definition of the quasi-local energy-momentum. The first expression that may be considered as a definition of the quasi-local energymomentum is probably that of Komar [34] (and its modification [35]). This associates an integral with any closed orientable two-surface and a vector field (the 'generator of the quasi-local quantity'). There are proposals based on more or less ad hoc ideas (but yielding useful energy or mass formulae, e.g. [36,37]), while others are based on Hamiltonian approaches of general relativity (see e.g. [38-41]).

The definition of Penrose [42] is based on the idea of charge integrals. This associates a (complex) integral with any closed orientable 2 -surface $\$$ and a symmetric spinor field $\omega^{A B}$ on it by

$$
\mathrm{A}_{\$}[\omega]:=\frac{\mathrm{i}}{\kappa} \oint_{\$} \omega^{A B} R_{A B c d} \mathrm{~d} x^{c} \wedge \mathrm{d} x^{d}
$$

The quasi-local energy-momentum and angular momentum might be expected to be such charge integrals with appropriately chosen 'generators' $\omega^{A B}$ for them. Penrose's original proposal was to consider $\omega_{A B}$ to be a combination $\omega_{\mathbf{a b}} \lambda_{A}^{\mathbf{a}} \lambda_{B}^{\mathbf{b}}$ of the symmetrized tensor products of the linearly independent solutions $\lambda_{A}^{\mathbf{a}}$ of the two-surface twistor equation by complex constants $\omega_{\mathbf{a b}}=\omega_{(\mathbf{a b})}$. With this choice $\mathrm{A}_{\$}[\omega]$ can be interpreted as the integral of a superpotential [43], too. Finally, many of the proposals in the spinorial approach are based on the two-surface integral of the Nester-Witten 2-form (1.3). The different constructions correspond to different chioces for the pair of spinor fields $\lambda \frac{A}{A}$ [44-47].

Since we would like to retain the four dimensional spirit of spacetime physics, we want not only quasi-local energy but a real four-vector $P_{\$}^{a}$ associated with a spacelike 
topological two-sphere (and mass as its length). We think that any physically acceptable quasi-local energy-momentum and angular momentum expression may depend only on the intrinsic and extrinsic geometry of $\$$ (and should not depend e.g. on the way how its normals are extended off $\$$ ). Furthermore we expect $P_{\$}^{a}$ to be interpretable as a Noether charge. These three requirements may be satisfied by an expression based on the integral (1.3) of the Nester-Witten 2-form. Having accepted (1.3) as a definition of the general framework for the energy-momentum the following basic question remains: How to choose the two spinor fields $\lambda_{A}^{0}, \lambda_{A}^{1} \in C^{\infty}\left(\$, \mathbf{S}_{A}\right)$ such that the Hermitian matrix $P_{\$} \underline{A} \underline{B}^{\prime}:=H_{\$}\left[\lambda \underline{A}, \bar{\lambda} \underline{B}^{\prime}\right]$ represents reasonable gravitational energy-momentum. Or, in other words, how to define the (spinor constituents of the) generator of the quasi-local energy-momentum, the so-called 'quasi-translations' of \$. Mathematically, we need a linear differential operator $\not D$, depending on the intrinsic and extrinsic geometry of $\$$, such that $\operatorname{dim} \operatorname{ker} \not D=2$ and the two spinor fields $\lambda_{A}^{A}$ are expected to span ker $\not D$. For example Dougan and Mason [46] chose holomorphic or anti-holomorphic spinor fields: $\bar{m}^{a} \nabla_{a} \lambda_{R}=0$ or $m^{a} \nabla_{a} \lambda_{R}=0$. Their choices, however, were justified a posteriori by the nice, acceptable properties of their energy-momentum expression [46,48-50]. Thus the question arises whether there are other possibilities or the Dougan-Mason choices are the 'natural' ones. Furthermore, one may asks how the different constructions (e.g. those based on charge integrals (1.6) and on the Nester-Witten integrals (1.3)) are related to each other. Obviously, it is a natural claim to clarify the properties of the 'most natural' quasi-local energy-momentum.

The usual formalism in the spinorial approaches is the elegant GHP formalism, which has been applied extensively to study the geometry of spacelike 2-surfaces [10,51]. Although it has some sophisticated form of covariance, a lot of experience is needed to 'see' the geometric content of the formulae in this formalism. The traditional covariance of a formalism may help and suggest why and what to calculate. Moreover, the extrinsic curvatures of $\$$ play important role in the present problems. Thus, to clarify the questions above, we want a covariant spinor formalism on the spacelike 2-surfaces in which the extrinsic curvatures are fit nicely. This lead us to the two dimensional Sen geometry of spacelike 2-surfaces $[52,53]$. From the study of the Sen geometry an interesting, unexpected relationship between the two dimensional Ashtekar connection, the Sen- and the 2 -surface twistor geometry appeared. The two dimensional Sen geometry was applied to determine the 'most natural' spinor propagation law $\not D \lambda_{R}=0$. Assuming that $\not D$ is first order and is built up only from the irreducible parts of the Sen operator, it was shown [54] that $\not D$ must be the holomorphy or anti-holomorphy operator of Dougan and Mason. Thus their choices have been supported. To clarify the properties of the Dougan-Mason energy-momentum further, we determined the zero energy-momentum and zero mass spacetime configurations [50]. It turned out, however, that these configurations can be characterized completely by constant spinor fields admitted by the two dimensional Sen geometry [54].

In Section 2 we review the main points of the two dimensional Sen geometry. We will see that this is not simply a copy of the three dimensional one in one fewer dimensions. An important difference between the one and two co-dimensional submanifolds is that while 
in the co-dimension one case the normal is uniquely determined, in the co-dimension two case there is a 1-parameter family of unit normals, and as a consequence of this 'boost-gauge freedom' the two dimensional Sen and spin connections always have 'normal' pieces as well, and the induced spin connection is precisely the two dimensional antiself-dual Ashtekar connection. Section 3 is devoted to the study of the Dougan-Mason energy-momentum. We recall the main idea how the $\not D$ operator is determined by the Sen connection, and then we characterize the zero mass and zero energy-momentum spacetime configurations. They are flat and $p p$-wave spacetime geometries with pure radiation, respectively. In Section 4 we show that the $p p$-wave Cauchy developments can be characterized completely not only by the usual Cauchy data on a three dimensional Cauchy surface $\Sigma$ but by the two dimensional Sen geometry of its boundary $\partial \Sigma$, too. Finally, in Section 5, we dicuss the possibility of finding the quasi-local representation of the unconstrained (i.e. radiative) degrees of freedom of general relativity.

2. The Sen geometry of two-surfaces. The usual way of introduction of a new concept in mathematics is to give a list of symbols and to define the meaning of them. The Sen geometry of two-surfaces can be defined in this abstract way. Although, for the sake of simplicity, we will introduce the two dimensional Sen geometry of a two-surface $\$$ from the imbedding of $\$$ in a four dimensional Lorentzian spacetime, first we list the main objects of this construction and explain their meaning very briefly. Thus by a two dimensional Sen geometry we mean a collection $\left(\$, \mathbf{S}^{A}(\$), \gamma_{B}^{A}, \vartheta_{a}^{A A^{\prime}}, \varepsilon_{A B}, \Delta_{a}\right)$, where $\$$ is an orientable 2-manifold, $\mathbf{S}^{A}(\$)$ is a complex vector bundle of rank two over $\$, \gamma^{A}{ }_{B}$ is a bundle automorphism leaving the base points fixed and satisfying $\gamma_{A}^{A}=0$ and $\gamma^{A}{ }_{B} \gamma^{B}{ }_{C}=\delta_{C}^{A}, \vartheta_{a}^{A A^{\prime}}$ is a certain imbedding of the complexified tangent bundle $T^{\mathbf{C}} \$$ of $\$$ into the product bundle $\mathbf{S}^{A}(\$) \otimes \overline{\mathbf{S}}^{A^{\prime}}(\$), \varepsilon_{A B}$ is a symplectic fibre metric and, finally, $\Delta_{a}$ is a certain covariant derivation on $\mathbf{S}^{A}(\$)$, the so-called two dimensional Sen connection, which annihilates $\varepsilon_{A B}$. Here $\gamma^{A}{ }_{B}$ defines some form of chirality on $\mathbf{S}^{A}(\$)$, playing the role of the $\gamma_{5}$ matrix, and $\vartheta_{a}^{A A^{\prime}}$ is the 2-surface soldering form, which maps $T^{\mathbf{C}} \$$ isomorphically onto a certain subbundle of $\mathbf{S}^{A}(\$) \otimes \overline{\mathbf{S}}^{A^{\prime}}(\$)$ defined by $\gamma^{A}{ }_{B}$.

The relativist's definition of the Sen geometry is much more constructive [52,53]: If $t^{a}, v^{a}$ are future directed unit timelike and unit spacelike normals of $\$$, respectively, such that $t_{a} v^{a}=0$, then $\Pi_{b}^{a}:=\delta_{b}^{a}-t^{a} t_{b}+v^{a} v_{b}$ is the projection of $T_{p} M$ onto $T_{p} \$$ for all $p \in \$$. By means of $\Pi_{b}^{a}$ one can define surface tensors, e.g. $q_{a b}:=\Pi_{a}^{e} \Pi_{b}^{f} g_{\text {ef }}$ is the induced two-metric on $\$$. Let $\mathbf{S}^{A}(\$)$ be the bundle of contravariant unprimed spinors on $\$$, and let us define $\gamma_{B}^{A}:=2 t^{A A^{\prime}} v_{B A^{\prime}} \cdot \gamma_{B}^{A}$ is invariant with respect to the 'boost-gauge transformation' $\left(t^{a}, v^{a}\right) \mapsto\left(t^{a} \cosh \eta+v^{a} \sinh \eta, t^{a} \sinh \eta+v^{a} \cosh \eta\right)$ and the conformal rescalings of the spacetime metric, and it turns out that all the algebraic properties of $\$$ are encoded in $\gamma^{A}{ }_{B}$. E.g. $\Pi_{b}^{a}=\frac{1}{2}\left(\delta_{B}^{A} \delta_{B^{\prime}}^{A^{\prime}}-\gamma^{A}{ }_{B} \bar{\gamma}^{A^{\prime}}{ }_{B^{\prime}}\right)$. Obviously $\gamma: \mathbf{S}^{A}(\$) \rightarrow \mathbf{S}^{A}(\$)$ : $\xi^{A} \mapsto \gamma^{A}{ }_{B} \xi^{B}$ is an automorphism of $\mathbf{S}^{A}(\$)$. As a consequence of the definition $\gamma^{A}{ }_{A}=0$ and $\gamma^{A}{ }_{B} \gamma^{B}{ }_{C}=\delta_{C}^{A}$ hold. Thus $\gamma_{B}^{A}$ has two eigenvalues, \pm 1 , and $\pi^{ \pm A}{ }_{B}:=\frac{1}{2}\left(\delta_{B}^{A} \pm \gamma^{A}{ }_{B}\right)$ are the projections of $\mathbf{S}^{A}(\$)$ to the bundle $\mathbf{S}^{A \pm}(\$)$ of the \pm 1 (right handed/left handed) eigenspinors, respectively. Thus $\gamma^{A}{ }_{B}$ defines a chirality on $\mathbf{S}^{A}(\$)$. If $o^{A}$ is a left handed and $\iota^{A}$ is a right handed spinor normalized by $o_{A} \iota^{A}=1$, then they form a GHP spinor dyad 
on $\$$. The restriction of the soldering form $\theta_{a}^{A A^{\prime}}: T^{\mathbf{C}} M \rightarrow \mathbf{S}^{A}(M) \otimes \overline{\mathbf{S}}^{A^{\prime}}(M)$ to $T^{\mathbf{C}} \$$, denoted by $\vartheta_{a}^{A A^{\prime}}$, maps $T^{\mathbf{C}} \$$ isomorphically to the Whitney-sum $\left(\mathbf{S}^{A-}(\$) \otimes \overline{\mathbf{S}}^{A^{\prime}+}(\$)\right)$

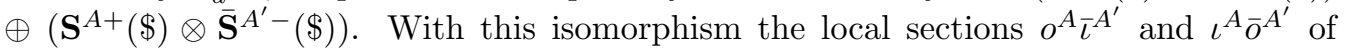
$\mathbf{S}^{A-}(\$) \otimes \overline{\mathbf{S}}^{A^{\prime}+}(\$)$ and $\mathbf{S}^{A+}(\$) \otimes \overline{\mathbf{S}}^{A^{\prime}-}(\$)$ become $(1,0)$ and $(0,1)$ type vector fields on $\$$, respectively, in the complex structure of $\$[55] . \gamma_{A B}=\gamma_{(A B)}=2 o_{\left(A^{\iota} \iota_{B}\right.}$ can also be considered as a complex fibre metric. Thus there are two metrics on the fibres, the symplectic $\varepsilon_{A B}$ and the symmetric $\gamma_{A B}$.

Let us define the two dimensional Sen operator by $\Delta_{a}:=\Pi_{a}^{b} \nabla_{b}$. The action of $\Delta_{a}$ is defined on every (and not only surface) tensor and spinor field, and obviously $\Delta_{e} \varepsilon_{A B}=0$. The two extrinsic curvatures of $\$, \tau_{a b}:=\Pi_{a}^{e} \Pi_{b}^{f} \nabla_{e} t_{f}$ and $\nu_{a b}:=\Pi_{a}^{e} \Pi_{b}^{f} \nabla_{e} v_{f}$, can be given in a boost-gauge invariant form by $Q_{a b}^{e}:=-\Pi_{f}^{e} \nabla_{a} \Pi_{b}^{f}=\tau_{a}^{e} t_{b}-\nu_{a}^{e} v_{b}$. Then $Q_{e a b}=Q_{(e a) b}$, and the expansion tensor of the out and ingoing null rays orthogonal to $\$$ are $\theta_{b}^{a}=Q_{b e}^{a} l^{e}$ and $\theta_{b}^{\prime a}=Q_{b e}^{a} n^{e}$, respectively, where $l^{a}$ and $n^{a}$ are the corresponding null normals. The mean curvatures of $\$$ are the traces $\theta^{e}{ }_{e}$ and $\theta^{\prime}{ }_{e}$, which are just -2 times the GHP spin coeffitients $\rho$ and $\rho^{\prime}$, respectively. Let us define $k:=\operatorname{det}\left\|\theta^{a}{ }_{b}\right\|=\frac{1}{2}\left(\theta_{a b} \theta_{c d}-\right.$ $\left.\theta_{a c} \theta_{b d}\right) q^{a b} q^{c d}$ and $k^{\prime}:=\operatorname{det}\left\|\theta^{\prime a}{ }_{b}\right\|=\frac{1}{2}\left(\theta_{a b}^{\prime} \theta_{c d}^{\prime}-\theta_{a c}^{\prime} \theta_{b d}^{\prime}\right) q^{a b} q^{c d}$. These are generalizations of the Gauss curvature known in the theory of surfaces. Note that although $\rho, \rho^{\prime}, k$ and $k^{\prime}$ are not boost gauge invariant, their signs are. $Q_{a b}^{e}$ can be reexpressed by the 'non- $\gamma_{A B}$-metricity' $Q^{A}{ }_{e B}:=\frac{1}{2} \Delta_{e} \gamma^{A}{ }_{R} \gamma^{R}{ }_{B}$ of the Sen operator as

$$
Q_{a b}^{e}=\frac{1}{2}\left(\delta_{B^{\prime}}^{E^{\prime}} Q_{a B}^{E}+\delta_{B}^{E} \bar{Q}_{a B^{\prime}}^{E^{\prime}}+Q_{a R}^{E} \gamma_{B}^{R} \bar{\gamma}_{B^{\prime}}^{E^{\prime}}+\bar{Q}_{a R^{\prime}}^{E^{\prime}} \bar{\gamma}_{B^{\prime}}^{R^{\prime}}{ }^{E}{ }_{B}\right)
$$

The action of the commutator of two Sen operators on a spinor field is

$$
\left(\Delta_{c} \Delta_{d}-\Delta_{d} \Delta_{c}\right) \xi^{A}=-R_{B e f}^{A} \Pi_{c}^{e} \Pi_{d}^{f} \xi^{B}-2 Q_{[c d]}^{e} \Delta_{e} \xi^{A} .
$$

Thus the curvature of the Sen operator is the pull back to $\$$ of the anti-self-dual part of the spacetime curvature: $F^{A}{ }_{B c d}:=R_{B e f}^{A} \Pi_{c}^{e} \Pi_{d}^{f}$, and $T^{e}{ }_{c d}:=2 Q^{e}{ }_{[c d]}$ plays the role of the torsion. By (2.1) $Q_{A E E^{\prime} B}$ is just the anti-self-dual part of the torsion: $T_{E E^{\prime} A A^{\prime} B B^{\prime}}=$ $-\left(\varepsilon_{A^{\prime} B^{\prime}} Q_{A E E^{\prime} B}+\varepsilon_{A B} \bar{Q}_{A^{\prime} E E^{\prime} B^{\prime}}\right)$. Expressing $R_{A B c d}$ by the Weyl and Ricci spinors and the $\Lambda$ scalar $F_{A B c d}$ can be reexpressed as

$$
F_{A B c d}=-\frac{\mathrm{i}}{2}\left(\psi_{A B E F} \gamma^{E F}-\phi_{A B E^{\prime} F^{\prime}} \bar{\gamma}^{E^{\prime} F^{\prime}}+2 \Lambda \gamma_{A B}\right) \varepsilon_{c d}
$$

Here $\varepsilon_{c d}:=t^{a} v^{b} \varepsilon_{a b c d}=\frac{\mathrm{i}}{2}\left(\gamma_{C D} \varepsilon_{C^{\prime} D^{\prime}}-\bar{\gamma}_{C^{\prime} D^{\prime}} \varepsilon_{C D}\right)$ is the induced volume 2 -form on $\$$.

The induced spin connection is defined by

$$
\delta_{e} \lambda^{A}:=\Delta_{e} \lambda^{A}-Q^{A}{ }_{e B} \lambda^{B},
$$

which for surface tensors is precisely the induced Levi-Civita covariant differentiation. $\delta_{e}$ annihilates both $\varepsilon_{A B}$ and $\gamma_{A B}$, and its curvature, defined by $-{ }^{\$} R^{A}{ }_{B c d} \xi^{B} X^{c} Y^{d}:=$ $X^{c} \delta_{c}\left(Y^{d} \delta_{d} \xi^{A}\right)-Y^{c} \delta_{c}\left(X^{d} \delta_{d} \xi^{A}\right)-[X, Y]^{e} \delta_{e} \xi^{A}$ for any spinor field $\xi^{A}$ and surface vector fields $X^{a}$ and $Y^{a}$, takes the form

$$
{ }^{\$} R_{A B c d}=-\frac{1}{2} \gamma_{A B}\left(\left(\delta_{c} A_{d}-\delta_{d} A_{c}\right)-\frac{\$}{4}\left(\varepsilon_{C^{\prime} D^{\prime}} \gamma_{C D}-\varepsilon_{C D} \bar{\gamma}_{C^{\prime} D^{\prime}}\right)\right) .
$$

Here $A_{e}:=\left(\Delta_{e} t_{f}\right) v^{f}$, which, under a boost-gauge transformation, transforms as an $S O(1,1)$-boost-gauge potential. With this extension of $\delta_{a}$ from surface tensors to spinors 
we have extended the Levi-Civita covariant derivation to arbitrary tensors. The 'normal' piece of this connection is represented by $A_{e}$.

Next let us consider ${ }^{ \pm} A_{e \underline{b}}^{\underline{a}}:=\prod_{e}^{f}\left(\omega_{f \underline{b}}^{\underline{a}} \mp \frac{i}{2} \varepsilon^{\underline{a}} \underline{b} \underline{c} \underline{d} \omega_{f}^{\underline{c}} \underline{d}\right)$, the pull back to $\$$ of the twice the self-dual (anti-self-dual) part of the spacetime connection [56]. Here underlined indices run from 0 to 3 and they refer to an orthonormal basis $\left\{E_{\underline{a}}^{a}\right\}$. If $\left\{E_{\underline{a}}^{a}\right\}$ is adapted to $\$$ in the sense e.g. that $E_{0}^{a}=t^{a}$ and $E_{3}^{a}=v^{a}$, then ${ }^{ \pm} A_{e}:=\frac{1}{2}^{ \pm} A_{e}^{a} \underline{a} \varepsilon_{a b}=E_{1}^{a} q_{a b} \delta_{e} E_{2}^{b} \pm \mathrm{i} A_{e}$; and, for $\underline{m}, \underline{n}, \ldots=1,2,{ }^{ \pm} A_{e} \frac{m}{e} \underline{n}={ }^{ \pm} A_{e} \varepsilon \underline{m} \underline{n}$ is the two dimensional version of the self-dual (anti-self-dual) Ashtekar connection. Then it is natural to define the two dimensional selfdual (anti-self-dual) Ashtekar covariant derivative of the (complex) tangent vector field $X^{a}=E_{m}^{a} X^{\underline{m}}$ by ${ }^{ \pm} \delta_{e} X^{a}:=E_{m}^{a}\left(\partial_{e} X^{\underline{m}}+{ }^{ \pm} A_{e} \varepsilon^{\underline{m}}{ }_{n} X^{\underline{n}}\right)=\delta_{e} X^{a} \pm \mathrm{i} A_{e} \varepsilon^{a}{ }_{b} X^{b}$. Thus, if $\varepsilon_{A}^{A}, \underline{A}=0,1$, is a GHP spinor dyad (whose elements are eigenspinors of $\gamma^{A}{ }_{B}$ ) then the action ${ }^{ \pm} \delta_{e}$ on $\lambda^{A}=\varepsilon_{\underline{A}}^{A} \lambda \underline{A}$ should be defined by ${ }^{ \pm} \delta_{e} \lambda^{A}:=\varepsilon_{\underline{A}}^{A}\left(\partial_{e} \lambda \underline{A}-\frac{\mathrm{i}}{2}{ }^{ \pm} A_{e} \gamma^{\underline{A}} \underline{B} \lambda^{\underline{B}}\right)$. A short calculation shows that our spin connection, defined by $(2.4)$, is precisely ${ }^{\underline{-}} \delta_{e}$.

Because of the presence of the extra, complex metric $\gamma_{A B}$ on the spin spaces, the decomposition of a two-index spinor $\phi_{A B}$ into its symmetric and anti-symmetric parts, $\phi_{(A B)}$ and $\frac{1}{2} \varepsilon_{A B} \phi_{R}{ }^{R}$, respectively, is not irreducible any more. The symmetric part can be decomposed further as the sum of its $\gamma_{A B}$-trace and its trace-free part. Similar decomposition can be carried out in the unprimed indices of the Sen-derivative of a spinor field $\lambda_{A}$ :

$$
\begin{aligned}
\Delta_{A^{\prime} A} \lambda_{B} & =\frac{1}{2} \varepsilon_{A B} \Delta_{A^{\prime} R} \lambda^{R}-\frac{1}{2} \gamma_{A B} \gamma^{R S} \Delta_{A^{\prime} R} \lambda_{S}+\mathcal{T}_{A^{\prime} A B}{ }^{R} \lambda_{R}= \\
& =\Pi_{A^{\prime}{ }_{A} \varepsilon_{R B}}^{R^{\prime}{ } \Delta_{R^{\prime} K} \lambda^{K}+\mathcal{T}_{A^{\prime} A B}{ }^{R} \lambda_{R} .}
\end{aligned}
$$

The equation $\Delta_{A^{\prime}{ }_{A}} \lambda^{A}=0$ would be analogous to the Sen-Witten equation, thus $\Delta_{A^{\prime}{ }_{A} \lambda^{A}}$ may be called the Sen-Witten part of $\Delta_{A A^{\prime}} \lambda_{B}$. The trace-free symmetric part $\mathcal{T}_{A^{\prime} A B}{ }^{R} \lambda_{R}$ turns out to be the two-surface twistor derivative of $\lambda_{R}$. E.g. the two-surface twistor equations [42,57] are equivalent to $\mathcal{T}_{A^{\prime} A B}{ }^{R} \lambda_{R}=0$, and $\mathrm{i} \Delta_{A^{\prime} A^{A}} \lambda^{A}$ is just the secondary part of a local twistor [57]. Finally, one can define the right/left handed parts of these operators in their primed indices:

$$
\begin{gathered}
\Delta_{R^{\prime}}^{ \pm}{ }^{R} \lambda_{R}:=\bar{\pi}^{\mp S^{\prime}}{ }_{R^{\prime}} \Delta_{S^{\prime}}{ }^{S} \lambda_{S}, \\
\mathcal{T}_{R^{\prime} R S}^{ \pm}{ }^{K} \lambda_{K}:=\bar{\pi}^{\mp K^{\prime}}{ }_{R^{\prime}} \mathcal{T}_{K^{\prime} R S}{ }^{K} \lambda_{K} .
\end{gathered}
$$

These four operators form the complete irreducible decomposition of the Sen operator in the sense that any further application of the symmetry operations and projections on them yields zero or acts as identity.

Finally, suppose that $\$$ is closed. Then, using the commutator (2.2) and the definitions, one can derive the two dimensional version of the (integrated) Sen-identity:

$$
\oint_{\$} \bar{\gamma}^{R^{\prime} S^{\prime}}\left(\Delta_{R^{\prime}}{ }^{R} \lambda_{R} \Delta_{S^{\prime}}{ }^{S} \omega_{S}+\mathcal{T}_{R^{\prime} R S}{ }^{K} \lambda_{K} \mathcal{T}_{S^{\prime}}{ }^{R S L} \omega_{L}\right) \mathrm{d} \$=-\frac{\mathrm{i}}{2} \oint_{\$} \lambda^{A} \omega^{B} R_{A B c d} \mathrm{~d} x^{c} \wedge \mathrm{d} x^{d}
$$

where $\mathrm{d} \$$ is the volume element on $\$$. The right hand side of $(2.8)$ is just Penrose's charge integral. If the spinor fields are solutions of the two-surface twistor equation then (2.8) reduces to Tod's formula expressing the charge integral in terms of the secondary parts of the two-surface twistors [57]. Furthermore, since in our formalism the integrand of 
(1.3) takes the form $\bar{\gamma}^{R^{\prime} S^{\prime}} \Delta_{R^{\prime}}{ }^{R} \lambda_{R} \bar{\mu}_{S^{\prime}}$, Penrose's charge integrals for the solutions of the two-surface twistor equation, and hence his energy-momentum expression, can be written as the Nester-Witten integral $H_{\$}\left[\lambda_{A}, \Delta_{A^{\prime} B} \omega^{B}\right]$.

3. The Dougan-Mason energy-momentum. Having accepted (1.3) as the framework in which the quasi-local energy-momentum is searched for, we must specify the spinor propagation law $\not D \lambda_{A}=0$ within $\$$; i.e. the linear differential operator $\not D$ : $C^{\infty}\left(\$, \mathbf{S}_{A}\right) \rightarrow E^{\infty}(\$)$, where $E^{\infty}(\$)$ is the space of the smooth sections of some vector bundle $E(\$)$ over $\$$. Since we would like to interpret the solutions of $\not D \lambda_{A}=0$ as the spinor constituents of the quasi-translations of $\$, \operatorname{dim}_{\mathbf{C}}$ ker $\not D=2$ is expected. Furthermore, if $\$$ is imbedded in the Minkowski spacetime then the quasi-translations defined according to the definition we are intending to introduce here are expected to coincide with the restriction of the familiar translational Killing vectors to $\$$. This, however, can be ensured only if $\not D$ depends on the extrinsic curvatures of $\$$, too. Thus, in the GHP formalism, $\not D$ is built up from the operators $\partial$ and $\partial^{\prime}$ and the spin coeffitients $\sigma, \rho, \sigma^{\prime}$ and $\rho^{\prime}$ (and perhaps from certain curvature components). However, without further idea these resrtictions do not determine $\not D$, too much freedom remain. On the other hand, the Sen operator contains all the information about the extrinsic curvatures of $\$$, since it is just the sum of the two dimensional spin connection and the (spinor form of the) extrinsic curvature. Thus, in principle, the Sen operator, or more precisely its chiral irreducible parts,

$$
\Delta^{+}, \Delta^{-}, \mathcal{T}^{+}, \mathcal{T}^{-}: E^{\infty}(-1,0) \oplus E^{\infty}(1,0) \simeq C^{\infty}\left(\$, \mathbf{S}_{A}\right) \rightarrow E^{\infty}(p, q),
$$

might be enough to determine $\not D$, where $E(m, n)$ is the bundle of $(m, n)$ type scalars over $\$[51], m, n \in \mathbf{R}$ such that $m-n \in \mathbf{Z}$, and the actual values $p, q$ depend on the chiral irreducible part considered. It turns out that this expectation is in fact correct [54], namely, if $\$$ is homeomorphic to $S^{2}$ then the only first order linear differential operators on $C^{\infty}\left(\$, \mathbf{S}_{A}\right)$ built up only from the chiral irreducible parts (3.1) of the Sen operator and whose kernel is generically two complex dimensional are $-\mathcal{H}^{+}:=\Delta^{+} \oplus \mathcal{T}^{+}$and $-\mathcal{H}^{-}:=$ $\Delta^{-} \oplus \mathcal{T}^{-}$, the holomorphy and anti-holomorphy operators. These are precisely the choices of Dougan and Mason [46]. The symplectic inner product of the certainly existing two holomorphic (anti-holomorphic) spinor fields is always constant, and in the generic case this is non-zero; i.e. the two holomorphic (anti-holomorphic) spinor fields span the spin space at each point of $\$$. If however this inner product is zero (these two-spheres are called 'exceptional two-spheres'), the two linearly independent solutions of $\not D \lambda_{A}=0$ do not form a pointwise linearly independent system of spinor fields on $\$$ and they cannot be interpreded as quasi-translations of $\$$. There might also be exceptional two-spheres for which there are more than two holomorphic or anti-holomorphic spinor fields. In these exceptional cases the Dougan-Mason energy-momentum is not well defined.

In what follows we assume that $\$$ is generic, and let $\lambda \frac{A}{A}, \underline{A}=0,1$, be two linearly independent anti-holomorphic spinor fields normalized by $\varepsilon^{A B} \lambda \frac{A}{A} \lambda \frac{B}{B}=\epsilon \underline{A} \underline{B}$. Then the the Dougan-Mason quasi-local energy-momentum and mass are

$$
P_{\$}^{\underline{A}} \underline{B}^{\prime}:=H_{\$}\left[\lambda \underline{A}, \bar{\lambda}^{\underline{B}^{\prime}}\right]
$$




$$
m_{\$}^{2}:=\epsilon_{\underline{A}} \underline{B} \underline{\epsilon}_{\underline{C}^{\prime} \underline{D}^{\prime}} P_{\$}^{\underline{A}} \underline{C}^{\prime} P_{\$}^{\underline{B}} \underline{D}^{\prime}=2\left(P_{\$}^{00^{\prime}} P_{\$}^{11^{\prime}}-P_{\$}^{01^{\prime}} P_{\$}^{10^{\prime}}\right),
$$

respectively. These expressions have a number of nice properties [46,48-50]:

i. $P_{\$}^{\underline{A}} \underline{B}^{\prime}=0$ in flat spacetime.

ii. $P_{\$}^{A} \underline{B}^{\prime}$ yields the correct results in the weak field approximation.

iii. For round spheres in spherically symmetric spacetimes $P_{\Phi}^{\underline{A}} \underline{B}^{\prime}$ yields the standard result of [58].

$i v$. For small spheres of radius $r, P_{\Phi}^{A} \underline{B}^{\prime}$ is $\frac{4}{3} \pi r^{3}$ times the energy-momentum vector of the matter, while in vacuum it is proportional to $r^{5}$ times the Bel-Robinson energy-momentum with positive factor of proportionality.

$v$. If $\$$ tends to a spherical cut $\$_{\infty}$ of spacelike infinity then $P_{\$}^{\underline{A}} \underline{B}^{\prime}$ tends to $P \frac{A}{A D M} \underline{B}^{\prime}\left[\$_{\infty}\right]$.

$v i$. If $\$$ tends to a spherical cut $\$_{\infty}$ of the future null infinity then $P_{\Phi} \underline{A} \underline{B}^{\prime}$ tends to $P \bar{A}_{B} \underline{B}^{\prime}\left[\$_{\infty}\right]$. At the same time the expression in which the holomorphic rather than the anti-holomorphic spinor fields are used tends to infinity in general, and tends to $P \bar{B}_{B} \underline{B}^{\prime}{ }^{\prime}\left[\$_{\infty}\right]$ in stationary spacetimes. This gives the correct Bondi-Sachs energymomentum at past null infinity.

vii. If $\Sigma$ is a smooth spacelike hypersurface with boundary $\$:=\partial \Sigma$ such that $\$$ is convex in the sense that the ingoing null normals of $\$$ are not diverging on $\$$ (i.e. the GHP spin coefficient $\rho^{\prime}$ is non-negative) and the dominant energy condition is satisfied on $\Sigma$ then $H_{\$}[\lambda, \bar{\lambda}] \geq 0$ for any $\lambda_{A} \in \operatorname{ker} \mathcal{T}^{-}$. This implies that $P_{\$}^{00^{\prime}} \geq 0, P_{\$}^{11^{\prime}} \geq 0$ and $m_{\$}^{2} \geq 0$; i.e. the Dougan-Mason energy-momentum is a future directed nonspacelike vector.

Thus the Dougan-Mason energy-momentum has several nice physical properties. One may ask, however, whether the converse of $i$. is true or not; and whether the vanishing of $m_{\$}^{2}$ implies the flatness, like in the case of the ADM and Bondi-Sachs masses, or not. If not, then what are the zero-mass spacetimes? The answer is given by the next two theorems $[50,54]$ :

TheOREM 1. Suppose that $\$=\partial \Sigma, \$$ is convex in the sense that $\rho^{\prime} \geq 0$ and the dominant energy condition is satisfied on the domain of dependence $D(\Sigma)$ of $\Sigma$. Then the following statements are equivalent:

1. $P_{\mathscr{S}}^{\underline{A}} \underline{B}^{\prime}=0$,

2. $D(\Sigma)$ is flat,

3. there exist two linearly independent spinor fields on $\$$ that are constant with respect to the two dimensional Sen connection.

TheOREM 2. Suppose that $\$=\partial \Sigma, \rho^{\prime} \geq 0$ and the dominant energy condition is satisfied on $D(\Sigma)$. Then the following statements are equivalent:

1. $m_{\$}^{2}=0$,

2. $D(\Sigma)$ is a pp-wave geometry and the matter is pure radiation (i.e. $D(\Sigma)$ admits a constant null vector field $L^{a}$ and $\left.T_{a b} L^{b}=0\right)$,

3. there exists a Sen-constant spinor field on $\$$. 
The equivalence of the first two statements means that the converse of property $i$. above is also true, but the vanishing of the mass does not imply the flatness of $D(\Sigma)$. Zero mass is the characteristic property of pure matter plus gravitational waves with special symmetry, and these results are in accordance with our physical picture. However, the equivalence of the second and third statments are surprising from the viewpoint of the standard initial value problem of general relativity. Namely, the information that the four dimensional $D(\Sigma)$ is flat or is a $p p$-wave geometry with pure radiation is completly encoded not only in the familiar initial data set on the three dimensional $\Sigma$, but in the Sen geometry of the two dimensional boundary of $\Sigma$, too. Thus gravity, together with matter fields satisfying the dominant energy condition, is a system which has some form of rigidity.

4. pp-wave geometry encoded in $\$$. Although the theorems of the previous section appear to characterize the zero energy-momentum and zero mass cases on equal footing, there is an important difference between these two. While in the zero energy-momentum case we can determine the metric of $D(\Sigma)$ from the Sen geometry of $\$$ by the first theorem, that is flat, in the zero mass case we can determine the class the metric of $D(\Sigma)$ belongs to. Thus one may ask whether all the information about the metric of $D(\Sigma)$ itself is encoded in the Sen geometry of $\$$. The answer is in the affirmative [59], and in the present section we sketch its proof.

In what follows we assume that the conditions of the second theorem are satisfied. Let $\lambda_{A}$ be a Sen-constant spinor field, $\mu_{A}$ be a non-constant anti-holomorphic and $\nu_{A}$ a nonconstant holomorphic spinor field on $\$$. Then these spinor fields are nowhere vanishing and can be normalized by $\lambda_{A} \mu^{A}=1$ and $\lambda_{A} \nu^{A}=1$. Thus $\left\{\lambda_{A}, \mu_{A}\right\}$ and $\left\{\lambda_{A}, \nu_{A}\right\}$ are normalized anti-holomorphic and holomorphic spin frames, respectively. Their deviation can be characterized by the globally defined complex function $\Phi:=\mu_{A} \nu^{A}$ since

$$
\left(\lambda_{A}, \nu_{A}\right)=\left(\lambda_{A}, \mu_{A}\right)\left(\begin{array}{cc}
1 & -\Phi \\
0 & 1
\end{array}\right) .
$$

Thus $\Phi$ is the only non-trivial component of the $S L(2, \mathbf{C})$ matrix connecting the holomorphic and anti-holomorphic spin frames. For a moment let us fix $\left(\$, \gamma_{A B}, \varepsilon_{A B}\right)$ and let $\lambda_{A}$ be a fixed nowhere vanishing spinor field on $\$$. Then it is not difficult to prove that there is a one-to-one correspondence between the functions $\Phi: \$ \rightarrow \mathbf{C}$ modulo constants and the gauge equivalence classes of Sen connections on $\$$ admitting $\lambda_{A}$ as a constant spinor field. Applying the commutator (2.2) e.g. to $\mu^{A}$ and using (2.3), we obtain $q^{a b} \delta_{a} \delta_{b} \Phi=\left(\psi_{A B C D} \gamma^{C D}-\phi_{A B C^{\prime} D^{\prime}} \bar{\gamma}^{C^{\prime} D^{\prime}}+2 \Lambda \gamma_{A B}\right) \mu^{A} \mu^{B}$. Thus $\Phi$ is a potential for the curvature.

By theorem 2 we know that the domain of dependence $D(\Sigma)$ is a $p p$-wave geometry. Then, at the points of $\overline{D(\Sigma)}, \psi_{A B C D}=\psi \lambda_{A} \lambda_{B} \lambda_{C} \lambda_{D}$ for some complex function $\psi$. (For the sake of simplicity let us concentrate only on the vacuum case, when $\phi_{A B A^{\prime} B^{\prime}}=0$ and $\Lambda=0$.) Substituting this expression into the spinor Bianchi identity we get $\lambda^{A} \nabla_{A A^{\prime}} \psi=0$; while substituting it into the potential equation we obtain $q^{a b} \delta_{a} \delta_{b} \Phi=\left.\psi\right|_{\$} \gamma_{A B} \lambda^{A} \lambda^{B}$. Therefore $\left.\psi\right|_{\$}$, the value of the only independent component of the spacetime curvature on $\$$, is determined by the Sen geometry of $\$$. On the other hand, we know that $L_{a}:=\lambda_{A} \bar{\lambda}_{A^{\prime}}$ 
is constant on $D(\Sigma)$, and hence it is a gradient: $L_{a}=\nabla_{a} u$ for some smooth $u: D(\Sigma) \rightarrow \mathbf{R}$. Let us define the level surfaces of the function $u$ by $S_{u}:=\{p \in \Sigma \mid u(p)=u\}$. The surfaces $S_{u}$ form a smooth foliation of $\Sigma$, furthermore by the Gauss equation it is easy to show that they are locally flat Riemann surfaces. Let $(\zeta, \bar{\zeta})$ be local holomorphic and antiholomorphic coordinates on $S_{u}$; i.e. $M^{a}:=\left(\frac{\partial}{\partial \zeta}\right)^{a}$ and $\bar{M}^{a}:=\left(\frac{\partial}{\partial \zeta}\right)^{a}$ are $(1,0)$ and $(0,1)$ type vector fields, respectively, in the complex structure of $S_{u}$. Then there is a uniquely defined spinor field $I^{A}$ on $\Sigma$ such that $\lambda_{A} I^{A}=1$ and $\lambda^{A} \bar{I}^{A^{\prime}}=M^{a}, I^{A} \bar{\lambda}^{A^{\prime}}=\bar{M}^{a}$ hold. Then contracting the spinor Bianchi identity with $\bar{I}^{A^{\prime}}$ we get $M^{a} \nabla_{a} \psi=0$; i.e. the function $\psi$ is anti-holomorphic on each Riemann surface $S_{u}$. Thus if $B_{u}:=\$ \cap S_{u}$, the boundary of the Riemann surfaces $S_{u}$, were nonempty and had simple structure then the value of $\psi$ on $\$$ would determine $\psi$ completely on the whole $\Sigma$ and hence on the whole $D(\Sigma)$, too. In general $B_{u}$ may have very complicated structure. However, a certain generalization of the usual convexity condition of the theory of surfaces rules out the strange boundary structure [59]:

Proposition. If $k>0$ and $k^{\prime}>0$ on $\$$, and $\rho \leq 0$ and $\rho^{\prime} \geq 0$ somewhere on $\$$, then $S_{u}$ is homeomorphic to $\mathbf{R}^{2}$ and $B_{u}$ is a single closed smooth curve in $\$$.

Therefore $q_{a b}, \Phi$ and $\gamma_{A B} \lambda^{A} \lambda^{B}$ determine $\psi$ on $D(\Sigma)$. To show that the Sen geometry determines the metric of $D(\Sigma)$ too, we should choose a coordinate system.

By the proposition above the complex coordinates $(\zeta, \bar{\zeta})$ are globally defined on $\Sigma$, and they can be extended in a natural way along the maximally extended integral curves of $L^{a}$. Similarly, we can extend the function $u$ along these integral curves too. Finally, let $v$ be the affine parameter of the null geodesic integral curves of $L^{a}$, measured from $\Sigma$. Then $(v, \zeta, \bar{\zeta}, u)$ is a coordinate system on the set of points of the integral curves of $L^{a}$ crossing $\Sigma$ somewhere. In these coordinates the metric takes the form $d s^{2}=$ $2 d v d u-2 d \zeta d \bar{\zeta}+2(G d \zeta+\bar{G} d \bar{\zeta}) d u+2 H d u^{2}$, where $H$ is a real and $G$ is a complex function of $\zeta, \bar{\zeta}$ and $u$; and by an approporiate allowed transformation $\zeta \mapsto \zeta^{\prime}:=\exp (\mathrm{i} a(u)) \zeta$ one can ensure that $G$ satisfies the condition $\frac{\partial G}{\partial \bar{\zeta}}-\frac{\partial \bar{G}}{\partial \zeta}=0$. In these coordinates Einstein's equations take the form $\frac{\partial}{\partial \zeta}\left(\frac{\partial H}{\partial \zeta}-\frac{\partial \bar{G}}{\partial u}\right)=0$ and $\frac{\partial}{\partial \zeta}\left(\frac{\partial H}{\partial \zeta}-\frac{\partial \bar{G}}{\partial u}\right)=\psi$. Their integrability condition is just $\frac{\partial \psi}{\partial \zeta}=0$. To see that the boundary conditions determine the solution of Einstein's equations completely, let us restrict the coordinates further. Observe that for any smooth function $V$ of $\zeta, \bar{\zeta}$ and $u$ the mapping $\mathcal{V}:(v, \zeta, \bar{\zeta}, u) \mapsto(v+V(\zeta, \bar{\zeta}, u), \zeta, \bar{\zeta}, u)$ is a smooth diffeomorphism of the domain of the coordinate system onto itself and such a transformation preserves the form of the line element with $H^{\prime}:=H+\frac{\partial V}{\partial u}, G^{\prime}:=G+\frac{\partial V}{\partial \zeta}$. This freedom can be used to reduce the number of unknown functions in the line element. The usual choice is that in which $G^{\prime}=0[60,61]$. For such a transformation, however, the 'generating function' $V$ would not vanish on $\$=\partial \Sigma$; i.e. $\mathcal{V}$ would deform the 2 -surface $\$$. Since $\$$ is fixed in our problem and the boundary data are given on $\$$, we should find a weaker coordinate condition such that the corresponding transformation $\mathcal{V}$ leaves $\$$ fixed. In fact, there exists a uniquely determined function $V(\zeta, \bar{\zeta}, u)$, vanishing on $\$$, such that $G^{\prime}$ is a holomorphic function of $\zeta$. Thus $G^{\prime}$ is determined by its value on $\$$, and by the Einstein equations, $\frac{\partial^{2} H^{\prime}}{\partial \zeta \partial \bar{\zeta}}=0$ and $\frac{\partial^{2} H^{\prime}}{\partial \bar{\zeta}^{2}}=\psi+\frac{\partial^{2} \bar{G}^{\prime}}{\partial u \partial \bar{\zeta}}, H^{\prime}$ is also uniquely determined by its value on $\$$. Finally, it is a direct calculation to check that the value 
of $H^{\prime}$ and $G^{\prime}$ on $\$$ is determined by the Sen geometry of $\$$ and the boost gauge defined by $\mathcal{V}(\Sigma)$ on $\$$.

5. Quasi-local radiative modes? In Minkowski spacetime the source free Maxwell and linear Einstein equations take the form of the linear zero-rest-mass-field-equations, whose solutions can always be written as superpositions of Fourier modes. If $\$$ is a spacelike convex two-surface homeomorphic to $S^{2}$, then each Fourier mode can be characterized completely by its value on $\$$. Thus the two transversal radiative modes of Maxwell and linear Einstein theory are encoded in certain fields on $\$$. In exact general relativity (without any Killing symmetry) the radiative modes are defined only at null infinity, where the field equations become linear $[6,62]$. From the point of view of radiative modes property $v i$. of the Dougan-Mason energy-momentum is especially interesting: In stationary spacetimes (i.e. in absence of radiation) the energy-momentum based on the holomorphic and anti-holomorphic spin frames give the same result, $P \frac{A}{B} \underline{B}^{\prime}{ }^{\prime}\left[\$_{\infty}\right]$. Thus it is the deviation of the holomorphic and anti-holomorphic structures that characterizes the presence of radiation. As a by-product of the investigations of the properties of the Dougan-Mason energy-momentum we obtained a similar result for the $p p$-wave configurations of exact general relativity, namely the (purely radiative) four-metric is encoded into the deviation of the holomorphic and anti-holomorphic structures of $\$$, again. In these special cases the field equations are linear, and the radiative modes, defined by the Fourier componenets, coincide with the unconstrained degrees of freedom. The deviation of the holomorphic and anti-holomorphic structures can however be defined for generic 2-spheres in generic spacetimes too, which might yield the possibility of finding a nice quasi-local representation of the unconstrained degrees of freedom.

\section{References}

[1] F.W. Hehl, On the energy tensor of spinning massive matter in classical field theory and general relativity, Rep. Math. Phys. 955 (1976).

[2] B.F. Schutz, R. Sorkin, Variational aspects of relativistic field theories with application to perfect fluid, Ann. Phys. (N.Y.) 1071 (1977).

[3] A. Trautman, Conservation laws in general relativity, in Gravitation: An Introduction to Current Research, ed. L. Witten, Wiley, New York 1962.

[4] A.J. Anderson, Principles of Relativity Physics, Ch. 13, Academic Press, New York, London 1967.

[5] J.N. Goldberg, Invariant transformations, conservation laws, energy-momentum, in General Relativity and Gravitation, vol 1., ed. A. Held, Plenum Press, New York 1980.

[6] R. Geroch, Asymptotic structure of spacetime, in Asymptotic Structure of Spacetime, ed. F.P. Esposito and L. Witten, Plenum Press, New York 1977.

[7] R. Beig, B.G. Schmidt, Einstein's equations near spatial infinity, Commun. Math. Phys. 8765 (1982).

[8] A. Ashtekar, J.D. Romano, Spatial infinity as a boundary of spacetime, Class. Quantum Grav. 91069 (1992). 
[9] R. Penrose, Zero rest-mass fields including gravitation: asymptotic behaviour, Proc. Roy. Soc. London A 284159 (1965).

[10] R. Penrose, W. Rindler, Spinors and Spacetime, vol 1, Cambridge Univ. Press, Cambridge 1982.

[11] P. Schoen, S.-T. Yau, On the proof of the positive mass conjecture in general relativity, Commun. Math. Phys. 6545 (1979).

[12] E. Witten, A new proof of the positive energy theorem, Commun. Math. Phys. 80381 (1981).

[13] J.M. Nester, A new gravitational energy expression with a simple positivity proof, Phys. Lett. 83 A 241 (1981).

[14] W. Israel, J.M. Nester, Positivity of the Bondi gravitational mass, Phys. Lett 85 A 259 (1981).

[15] M. Ludvigsen, J.A.G. Vickers, The positivity of the Bondi mass, J. Phys. A.: Math. Gen. 14 L389 (1981).

[16] M. Ludvigsen, J.A.G. Vickers, A simple proof of the positivity of the Bondi mass, J. Phys. A.: Math. Gen. 15 L67 (1982).

[17] G.T. Horowitz, M.J. Perry, Gravitational energy cannot become negative, Phys. Rev. Lett. 48371 (1982).

[18] A. Ashtekar, G.T. Horowitz, Energy-momentum of isolated systems cannot be null, Phys. Lett. 89A 181 (1982).

[19] T. Parker, C.H. Taubes, On Witten's proof of the positive energy theorem, Commun. Math. Phys. 84223 (1982).

[20] O. Reula, Existence theorem for solutions of Witten's equation and nonnegativity of total mass, J. Math. Phys. 23810 (1982).

[21] G.T. Horowitz, K.P. Tod, A relation between local and total energy in general relativity, Commun. Math. Phys. 85429 (1982).

[22] O. Reula, K.P. Tod, Positivity of the Bondi energy, J. Math. Phys. 251004 (1984).

[23] G.T. Horowitz, The positive energy theorem and its extensions, in Asymptotic Behaviour of Mass and Spacetime Geometry, Lecture Notes in Physics 202, Ed.: F.J. Flaherty, Springer, New York, 1984.

[24] P.F. Yip, A strictly-positive mass theorem, Commun. Math. Phys. 108653 (1987).

[25] J. Jezierski, J. Kijowski, Positivity of total energy in general relativity, Phys. Rev. D 361041 (1987).

[26] J.M. Nester, A positive gravitational energy proof, Phys. Lett. 139 A 112 (1989).

[27] J.M. Nester, Positive energy via the teleparallel Hamiltonian, Int. J. Mod. Phys. A 4 1755 (1989).

[28] A. Dimakis, F. Müller-Hoissen, Spinor fields and the positive energy theorem, Class. Quantum Grav. 7283 (1990).

[29] G. Bergqvist, Simplified spinorial proof of the positive energy theorem, Phys. Rev. D 48628 (1993).

[30] L.J. Mason, J. Frauendiener, The Sparling 3-form, Ashtekar variables and quasi-local mass, in Twistors in Mathematics and Physics, ed. R. Baston and T. Bailey, London Math. Soc. Lecture Note Series, Cambridge Univ. Press, Cambridge 1990.

[31] J. Frauendiener, Geometric description of energy-momentum psoudotensors, Class. Quantum Grav. 6 L237 (1989).

[32] L.B.Szabados, On canonical pseudotensors, Sparling's form and Noether currents, Class. Quantum Grav. 92521 (1992), and KFKI Report 1991-29/B. 
[33] A. Sen, On the existence of neutrino 'zero-modes' in vacuum spacetimes J. Math. Phys. 221781 (1981).

[34] A. Komar, Covariant conservation laws in general relativity, Phys. Rev. 113934 (1959).

[35] J. Winicour, L. Tamburino, Lorentz-covariant gravitational energy-momentum linkages, Phys. Rev. Lett. 15601 (1965).

[36] S.W. Hawking, Gravitational radiation in an expanding universe, J. Math. Phys. 9598 (1968).

[37] R. Bartnik, New definition of quasilocal mass, Phys. Rev. Lett. 622346 (1989).

[38] J.D. Brown, J.M. York, Jr, Quasi-local energy and conserved charges derived from the gravitational action, Phys. Rev. D 471407 (1993).

[39] S. Lau, Canonical variables and quasi-local energy in general relativity, Class. Quantum Grav. 102379 (1993).

[40] S.A. Hayward, Quasilocal gravitational energy, Phys. Rev. D 49831 (1994).

[41] J. Kijowski, A simple derivation of canonical structure and quasi-local Hamiltonians in general relativity, (unpublished) 1995.

[42] R. Penrose, Quasi-local mass and angular momentum in general relativity, Proc. Roy. Soc. Lond. A381 53 (1982).

[43] J.N. Goldberg, Conserved quantities at spatial and null infinity: The Penrose potential, Phys. Rev. D 4141 (1990).

[44] M. Ludvigsen, J.A.G. Vickers, Momentum, angular momentum and their quasi-local null surface extensions, J. Phys. A: Math. Gen. 161155 (1983).

[45] G. Bergqvist, M. Ludvigsen, Quasilocal momentum and angular momentum in Kerr spacetime, Class. Quantum Grav. 8697 (1991).

[46] A.J. Dougan, L.J. Mason, Quasilocal mass constructions with positive energy, Phys. Rev. Lett. 672119 (1991).

[47] G. Bergqvist, Positivity and definitions of mass, Class. Quantum Grav. 91917 (1992).

[48] G. Bergqvist, Quasilocal mass for event horizons, Class. Quantum Grav. 91753 (1992).

[49] A.J. Dougan, Quasi-local mass for spheres, Class. Quantum Grav. 92461 (1992).

[50] L.B. Szabados, On the positivity of the quasi-local mass, Class. Quantum Grav. 101899 (1993).

[51] R. Geroch, A. Held, R. Penrose, A spacetime calculus based on pairs of null directions, J. Math. Phys. 14874 (1973).

[52] L.B. Szabados, Two dimensional Sen connections, in Relativity Today, Proceedings of the 4th Hungarian Relativity Workshop, Ed.: R.P. Kerr, Z. Perjés, Akadémiai Kiadó, Budapest 1993

[53] L.B. Szabados, Two dimensional Sen connections in general relativity, Class. Quantum Grav. 111833 (1994).

[54] L.B. Szabados, Two dimensional Sen connections and quasi-local energy-momentum, Class Quantum Grav. 111847 (1994).

[55] S. Kobayashi, K. Nomizu, Foundation of differential geometry, vol 2, Interscience, New York, 1968.

[56] A. Ashtekar, Lectures on Non-perturbative Canonical Gravity, World Scientific, Singapore, 1991.

[57] R. Penrose, W. Rindler, Spinors and Spacetime, vol 2, Cambridge Univ. Press, Cambridge, 1986 .

[58] C.W. Misner, D.H. Sharp, Relativistic equations for adiabatic, spherically symmetric gravitational collapse, Phys. Rev. 136 B571 (1964). 
[59] L.B. Szabados, Quasi-local energy-momentum and two-surface characterization of the pp-wave spacetimes, Class. Quantum Grav. 131661 (1996).

[60] D. Kramer, H. Stephani, M.A.H. Mac Callum, E. Herlt, Exact Solutions of Einstein's Field Equations, Cambridge Univ. Press, Cambridge 1980.

[61] P.C. Aichelburg, Remark on the superposition principle for gravitational waves, Acta Phys. Austriaca 34279 (1971).

[62] A. Ashtekar, Asymptotic Quantization, Bibliopolis, Naples 1987. 